\title{
Ancient olive trees as a source of olive oils rich in phenolic compounds
}

\author{
Nuno Rodrigues ${ }^{\mathrm{a}, \mathrm{b}}$, Susana Casal $^{\mathrm{c}}$, Teresa Pinho ${ }^{\mathrm{c}}$, António M. Peres ${ }^{\mathrm{a}, \mathrm{d}}$, Albino Bento ${ }^{\mathrm{a}}$, \\ Paula Baptista ${ }^{\mathrm{a}}$, José Alberto Pereira ${ }^{\mathrm{a}, *}$ \\ a Centro de Investigação de Montanha (CIMO), ESA, Instituto Politécnico de Bragança, Campus de Santa Apolónia, 5300-253 Bragança, Portugal \\ ${ }^{\mathrm{b}}$ Universidad de Léon, Departamento de Ingeniería Agrária, Av. Portugal, $n^{\circ}$ 41, 24071 Léon, Spain \\ c LAQV/REQUIMTE, Laboratory of Bromatology and Hydrology, Faculty of Pharmacy, University of Porto, Rua de Jorge Viterbo Ferreira, 228, 4050-313 Porto, Portugal \\ d Laboratory of Separation and Reaction Engineering - Laboratory of Catalysis and Materials (LSRE-LCM), ESA, Instituto Politécnico de Bragança, Campus Santa \\ Apolónia, 5300-253 Bragança, Portugal
}

\section{A R T I C L E IN F O}

\section{Keywords:}

Phenolic compounds

Health claims

Crop year

Olive heritage

\begin{abstract}
A B S T R A C T
Olive oil phenolic compounds are receiving increased attention due to its influence on sensory characteristics and to scientific evidences of positive health effects. In this work, 28 ancient olive trees were selected and, during four consecutive seasons (2014-2017), oils were extracted and their phenolic fraction characterized. Hydroxytyrosol and tyrosol secoiridoids were the predominant groups, with contents between 32 and $496 \mathrm{mg}$ of tyrosol equivalents $/ \mathrm{kg}$. Based on principal component analysis it could be concluded that the individual phenolic contents enabled the unsupervised grouping of olive oils by crop year. Furthermore, linear discriminant analysis allowed achieving sensitivities greater than $90 \%$. It was shown that some specimens consistently allowed obtaining oils with high phenolic contents ( $\geq 500 \mathrm{mg}$ tyrosol equivalents $/ \mathrm{kg}$ ). The identification of centenarian specimens for breeding based on their potential to produce oils with high levels of healthy compounds is of utmost interest, contributing to preserve the genetic heritage.
\end{abstract}

\section{Introduction}

Olive oil is one of the most important components of the Mediterranean diet, due to its organoleptic characteristics, nutritional properties, and cultural influences (García-Vico, García-Rodríguez, Sanz, \& Pérez, 2017; Fernández, Vidal, \& Canals, 2018; Polari, GarcíAguirre, Olmo-García, Carrasco-Pancorbo, \& Wanga, 2018). Compared to other vegetable oils, olive oils greatest richness is mainly due to differences in the production process (Reboredo-Rodríguez et al., 2017). Olive oils are extracted from fresh olives only by mechanical and physical processes (milling, malaxation and centrifugation), which allows keeping intact the properties of the fruit, especially compounds related to its bioactive capacity (phenolics, tocopherols, sterols, pigments, etc.) (Tsimidou \& Boskou, 2015; Visioli \& Bernardini, 2013).

The biological benefits of health due to olive oil consumption are not only related to the high monounsaturated fat content. Indeed, several minor components also have important bioactive properties contributing to its nutritional value (Khymenets et al., 2011). Among these, polyphenols have recently received great attention (Khoddami, Wilkes, \& Roberts, 2013; Shahidi \& Ambigaipalan, 2015), as they play a key role in human health, through its protective effects against neurodegenerative and cardiovascular diseases (Olmo-García, Bajoub,
Monasterio, Fernández-Gutiérrez \& Carrasco-Pancorbo, 2017; Visioli, 2012), while protecting the body from oxidative damage (Cicerale, Lucas, \& Keast, 2010; García-Rodríguez, Romero-Segura, Sanz, \& Pérez, 2015). Different works have demonstrated the positive correlations between the daily intake of phenolic compounds in the Mediterranean diet and health (Aparicio-Ruiz et al., 2016; Fregapane \& Salvador, 2013; Vitaglione et al., 2015). Recently, EFSA (European Food Safety Authority) recognized an health claim associated with the contribution of "olive oil polyphenols" for the protection of blood lipids from oxidative stress, which is only allowed for "olive oils containing at least $5 \mathrm{mg}$ of hydroxytyrosol and its derivatives (e.g. oleuropein complex and tyrosol) per $20 \mathrm{~g}$ of olive oil" (Commission Regulation (EU) 432/2012; EFSA, 2012). This official recognition has increased the interest on the subject, particularly by the olive oil industry, aiming to produce olive oils that could hold that claim (Reboredo-Rodríguez et al., 2017; Veneziani et al., 2018). Different polyphenols can be found in virgin olive oils, mostly derivatives of tyrosol, hydroxytyrosol, 4-hydroxybenzoic acid and 4-hydroxyphenylacetic acid, together with lignans, secoiridoids and flavonoids (García-Villalba et al., 2010). Although all are classified as phenolic compounds, not all are included in the above mentioned health claim. Oleuropein aglycon derivatives (dialdehyde and aldehyde forms) are usually the main phenolic compound in olives, derived from

\footnotetext{
* Corresponding author.

E-mail address: jpereira@ipb.pt (J.A. Pereira).
} 
Table 1

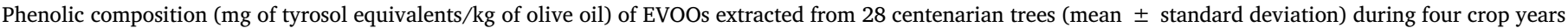
(2014-2017). A-Phenolic alcohols, Flavonoids and Secoiridoid Aglycons. B-Phenolic acids, Dihydroxybenzoic acid and Total Phenols.

\begin{tabular}{|c|c|c|c|c|c|c|c|}
\hline \multirow[t]{2}{*}{ A - Tree } & \multicolumn{2}{|l|}{ Phenolic alcohols } & \multicolumn{3}{|l|}{ Flavonoids } & \multicolumn{2}{|l|}{ Secoiridoids Aglycons } \\
\hline & Hydroxytyrosol & Tyrosol & Luteolin & Apigenin & Methyl luteolin & Oleuropein derivatives & Ligstroside derivatives \\
\hline Tree 1 & $3.7 \pm 2.7$ & $5.5 \pm 4.0$ & $8.1 \pm 3.7$ & $15.4 \pm 6.0$ & $2.0 \pm 1.0$ & $120.6 \pm 58.2$ & $270.9 \pm 100.4$ \\
\hline Tree 2 & $2.1 \pm 0.9$ & $3.2 \pm 1.8$ & $8.8 \pm 3.4$ & $6.7 \pm 2.2$ & $2.2 \pm 1.5$ & $104.8 \pm 25.4$ & $138.9 \pm 69.0$ \\
\hline Tree 3 & $2.7 \pm 1.3$ & $5.8 \pm 3.7$ & $12.9 \pm 4.0$ & $11.3 \pm 6.4$ & $2.5 \pm 1.3$ & $97.6 \pm 49.2$ & $229.6 \pm 175.6$ \\
\hline Tree 4 & $2.2 \pm 1.0$ & $1.9 \pm 1.0$ & $3.0 \pm 2.0$ & $3.0 \pm 2.3$ & $0.6 \pm 0.7$ & $116.1 \pm 19.4$ & $219.2 \pm 105.6$ \\
\hline Tree 5 & $2.0 \pm 1.0$ & $1.8 \pm 0.9$ & $2.6 \pm 1.4$ & $2.7 \pm 1.6$ & $0.8 \pm 1.0$ & $102.4 \pm 13.8$ & $212.0 \pm 106.6$ \\
\hline Tree 6 & $4.5 \pm 2.0$ & $6.6 \pm 2.0$ & $7.3 \pm 1.0$ & $8.0 \pm 1.8$ & $0.5 \pm 0.6$ & $117.3 \pm 11.2$ & $126.7 \pm 5.3$ \\
\hline Tree 7 & $1.9 \pm 1.3$ & $1.7 \pm 1.0$ & $2.3 \pm 0.7$ & $3.3 \pm 1.7$ & $0.7 \pm 0.8$ & $103.6 \pm 36.3$ & $191.6 \pm 47.2$ \\
\hline Tree 8 & $3.8 \pm 2.5$ & $4.9 \pm 3.7$ & $5.6 \pm 1.4$ & $13.7 \pm 12.1$ & $1.5 \pm 1.8$ & $119.7 \pm 60.1$ & $335.6 \pm 111.5$ \\
\hline Tree 9 & $1.9 \pm 1.1$ & $1.5 \pm 0.6$ & $2.5 \pm 0.3$ & $4.2 \pm 3.3$ & $2.2 \pm 2.8$ & $62.5 \pm 35.8$ & $185.2 \pm 99.7$ \\
\hline Tree 10 & $1.8 \pm 0.9$ & $1.6 \pm 0.7$ & $2.0 \pm 0.3$ & $2.3 \pm 1.2$ & $1.0 \pm 1.2$ & $94.0 \pm 10.9$ & $192.4 \pm 98.8$ \\
\hline Tree 11 & $2.1 \pm 0.5$ & $3.5 \pm 2.0$ & $4.6 \pm 1.8$ & $3.4 \pm 1.3$ & $0.7 \pm 0.5$ & $139.9 \pm 11.3$ & $216.7 \pm 116.0$ \\
\hline Tree 12 & $1.9 \pm 0.8$ & $4.8 \pm 2.0$ & $12.5 \pm 2.9$ & $15.3 \pm 4.2$ & $1.1 \pm 0.9$ & $124.5 \pm 33.3$ & $202.5 \pm 63.7$ \\
\hline Tree 13 & $1.9 \pm 1.0$ & $5.0 \pm 2.9$ & $14.9 \pm 3.2$ & $16.5 \pm 6.8$ & $13.6 \pm 0.8$ & $171.7 \pm 16.6$ & $190.8 \pm 32.8$ \\
\hline Tree 14 & $2.2 \pm 1.2$ & $2.2 \pm 1.5$ & $4.3 \pm 1.0$ & $4.9 \pm 4.0$ & $0.9 \pm 0.7$ & $134.4 \pm 73.4$ & $299.0 \pm 137.7$ \\
\hline Tree 15 & $1.6 \pm 0.4$ & $1.5 \pm 0.3$ & $2.0 \pm 0.5$ & $2.7 \pm 1.2$ & $0.9 \pm 1.1$ & $104.9 \pm 10.0$ & $197.6 \pm 100.5$ \\
\hline Tree 16 & $2.3 \pm 1.1$ & $4.7 \pm 2.9$ & $13.4 \pm 6.1$ & $15.3 \pm 9.0$ & $1.0 \pm 1.0$ & $151.8 \pm 38.5$ & $232.8 \pm 48.6$ \\
\hline Tree 17 & $6.8 \pm 3.9$ & $6.0 \pm 3.4$ & $6.2 \pm 3.0$ & $10.0 \pm 5.8$ & $0.7 \pm 1.2$ & $71.3 \pm 54.4$ & $198.9 \pm 137.1$ \\
\hline Tree 18 & $2.6 \pm 0.8$ & $2.0 \pm 0.3$ & $2.0 \pm 0.6$ & $2.8 \pm 1.2$ & $1.0 \pm 1.1$ & $104.3 \pm 29.9$ & $215.2 \pm 82.7$ \\
\hline Tree 19 & $2.6 \pm 1.0$ & $1.8 \pm 0.9$ & $11.0 \pm 16.0$ & $3.6 \pm 2.4$ & $1.1 \pm 1.2$ & $121.6 \pm 50.8$ & $219.5 \pm 81.0$ \\
\hline Tree 20 & $2.6 \pm 1.3$ & $2.0 \pm 0.6$ & $2.0 \pm 0.3$ & $2.8 \pm 1.9$ & $0.8 \pm 0.6$ & $79.8 \pm 53.3$ & $223.4 \pm 87.6$ \\
\hline Tree 21 & $3.2 \pm 1.7$ & $4.7 \pm 2.8$ & $9.5 \pm 4.1$ & $9.3 \pm 6.8$ & $0.6 \pm 0.6$ & $148.0 \pm 66.8$ & $219.8 \pm 45.1$ \\
\hline Tree 22 & $3.5 \pm 3.2$ & $5.9 \pm 4.8$ & $4.8 \pm 0.5$ & $6.4 \pm 4.5$ & $1.2 \pm 0.6$ & $61.9 \pm 46.1$ & $219.0 \pm 45.4$ \\
\hline Tree 23 & $5.4 \pm 5.2$ & $5.8 \pm 4.0$ & $12.0 \pm 4.5$ & $9.3 \pm 5.4$ & $1.3 \pm 1.0$ & $127.9 \pm 66.8$ & $256.4 \pm 118.5$ \\
\hline Tree 24 & $2.7 \pm 1.6$ & $4.6 \pm 2.3$ & $7.6 \pm 6.3$ & $7.4 \pm 8.2$ & $0.8 \pm 0.7$ & $249.6 \pm 133.6$ & $379.5 \pm 37.7$ \\
\hline Tree 25 & $4.6 \pm 3.4$ & $9.8 \pm 7.1$ & $4.9 \pm 1.5$ & $5.5 \pm 4.0$ & $0.3 \pm 0.3$ & $112.6 \pm 69.2$ & $410.8 \pm 164.6$ \\
\hline Tree 26 & $7.1 \pm 8.0$ & $12.1 \pm 9.3$ & $5.6 \pm 1.0$ & $7.0 \pm 5.5$ & $0.4 \pm 0.6$ & $129.9 \pm 92.3$ & $426.1 \pm 142.3$ \\
\hline Tree 27 & $1.1 \pm 0.9$ & $4.2 \pm 2.5$ & $1.7 \pm 0.6$ & $3.1 \pm 2.9$ & $0.1 \pm 0.2$ & $32.0 \pm 35.2$ & $171.5 \pm 73.3$ \\
\hline Tree 28 & $3.0 \pm 2.4$ & $3.9 \pm 2.2$ & $16.2 \pm 7.1$ & $12.1 \pm 7.4$ & $2.2 \pm 1.5$ & $247.1 \pm 94.0$ & $295.7 \pm 44.4$ \\
\hline Median & 2.3 & 2.5 & 4.6 & 4.8 & 0.7 & 109.8 & 201.1 \\
\hline Minimum & 0.0 & 0.3 & 0.8 & 0.6 & 0.0 & 1.8 & 85.3 \\
\hline Maximum & 20.0 & 26.4 & 37.3 & 31.6 & 6.8 & 414.1 & 591.4 \\
\hline \multirow[t]{2}{*}{ B - Tree } & \multicolumn{4}{|l|}{ Phenolic acids } & \multicolumn{2}{|c|}{ Dihydroxybenzoic derivatives } & Total Phenols \\
\hline & $p$-Coumaric acid & Ferulic acid & $o$-Coumaric acid & Cinnamic acid & Vanillic acid & Vanillin & \\
\hline Tree 1 & $3.3 \pm 2.3$ & $0.7 \pm 0.6$ & $0.4 \pm 0.4$ & $4.0 \pm 4.1$ & $3.4 \pm 1.8$ & $2.7 \pm 2.4$ & $441 \pm 90$ \\
\hline Tree 2 & $3.5 \pm 3.2$ & $0.3 \pm 0.2$ & $0.4 \pm 0.4$ & $2.1 \pm 1.5$ & $4.4 \pm 2.6$ & $1.7 \pm 1.7$ & $279 \pm 98$ \\
\hline Tree 3 & $2.8 \pm 1.5$ & $0.4 \pm 0.3$ & $0.3 \pm 0.2$ & $2.6 \pm 2.6$ & $4.8 \pm 2.8$ & $2.4 \pm 1.9$ & $376 \pm 142$ \\
\hline Tree 4 & $5.1 \pm 4.7$ & $0.6 \pm 0.3$ & $0.2 \pm 0.1$ & $2.2 \pm 2.2$ & $2.2 \pm 1.6$ & $2.0 \pm 1.9$ & $359 \pm 104$ \\
\hline Tree 5 & $3.1 \pm 1.1$ & $0.5 \pm 0.2$ & $0.3 \pm 0.3$ & $1.9 \pm 1.9$ & $2.4 \pm 2.0$ & $2.0 \pm 1.6$ & $335 \pm 110$ \\
\hline Tree 6 & $3.4 \pm 2.6$ & $0.3 \pm 0.0$ & $0.3 \pm 0.4$ & $1.9 \pm 2.0$ & $6.3 \pm 3.6$ & $2.2 \pm 0.6$ & $285 \pm 4$ \\
\hline Tree 7 & $4.5 \pm 3.6$ & $0.6 \pm 0.2$ & $0.4 \pm 0.5$ & $2.5 \pm 2.4$ & $2.7 \pm 2.0$ & $2.2 \pm 1.9$ & $318 \pm 39$ \\
\hline Tree 8 & $2.0 \pm 1.2$ & $0.5 \pm 0.3$ & $0.4 \pm 0.4$ & $4.1 \pm 4.2$ & $2.4 \pm 1.5$ & $2.0 \pm 1.5$ & $496 \pm 100$ \\
\hline Tree 9 & $3.2 \pm 2.2$ & $0.7 \pm 0.3$ & $0.5 \pm 0.7$ & $2.0 \pm 2.0$ & $3.1 \pm 1.6$ & $1.8 \pm 1.7$ & $271 \pm 82$ \\
\hline Tree 10 & $4.1 \pm 2.4$ & $0.7 \pm 0.2$ & $0.3 \pm 0.5$ & $2.1 \pm 2.1$ & $2.4 \pm 1.6$ & $2.0 \pm 1.8$ & $307 \pm 105$ \\
\hline Tree 11 & $10.8 \pm 8.1$ & $0.6 \pm 0.2$ & $0.6 \pm 0.8$ & $5.0 \pm 3.7$ & $4.1 \pm 2.9$ & $1.4 \pm 1.2$ & $393 \pm 100$ \\
\hline Tree 12 & $4.0 \pm 2.8$ & $0.4 \pm 0.2$ & $0.3 \pm 0.5$ & $3.7 \pm 3.7$ & $3.7 \pm 2.2$ & $1.6 \pm 1.4$ & $376 \pm 52$ \\
\hline Tree 13 & $4.1 \pm 2.8$ & $0.4 \pm 0.2$ & $0.4 \pm 0.6$ & $5.8 \pm 4.4$ & $3.7 \pm 2.3$ & $1.2 \pm 1.3$ & $418 \pm 64$ \\
\hline Tree 14 & $4.4 \pm 2.5$ & $0.6 \pm 0.2$ & $0.4 \pm 0.6$ & $3.9 \pm 4.5$ & $1.7 \pm 0.7$ & $1.9 \pm 1.6$ & $461 \pm 165$ \\
\hline Tree 15 & $3.7 \pm 1.7$ & $0.5 \pm 0.1$ & $0.3 \pm 0.5$ & $1.9 \pm 2.1$ & $2.3 \pm 1.2$ & $2.0 \pm 1.8$ & $322 \pm 103$ \\
\hline Tree 16 & $3.4 \pm 2.0$ & $0.5 \pm 0.3$ & $0.3 \pm 0.4$ & $4.9 \pm 4.8$ & $3.2 \pm 2.1$ & $1.6 \pm 1.4$ & $435 \pm 43$ \\
\hline Tree 17 & $2.1 \pm 1.4$ & $0.5 \pm 0.3$ & $1.9 \pm 2.0$ & $2.7 \pm 3.1$ & $4.3 \pm 2.8$ & $1.8 \pm 1.6$ & $313 \pm 168$ \\
\hline Tree 18 & $2.9 \pm 1.7$ & $0.6 \pm 0.1$ & $0.3 \pm 0.4$ & $2.5 \pm 2.7$ & $2.6 \pm 1.4$ & $2.1 \pm 1.8$ & $340 \pm 53$ \\
\hline Tree 19 & $5.6 \pm 3.9$ & $0.6 \pm 0.1$ & $0.3 \pm 0.5$ & $3.1 \pm 3.2$ & $2.2 \pm 1.3$ & $1.9 \pm 1.5$ & $375 \pm 79$ \\
\hline Tree 20 & $7.0 \pm 6.4$ & $0.7 \pm 0.2$ & $0.5 \pm 0.6$ & $2.7 \pm 2.9$ & $2.5 \pm 1.4$ & $2.4 \pm 2.2$ & $356 \pm 84$ \\
\hline Tree 21 & $2.6 \pm 2.0$ & $0.5 \pm 0.3$ & $0.8 \pm 0.5$ & $7.1 \pm 6.4$ & $3.2 \pm 2.0$ & $1.0 \pm 1.1$ & $410 \pm 133$ \\
\hline Tree 22 & $2.8 \pm 1.7$ & $0.5 \pm 0.3$ & $0.3 \pm 0.3$ & $4.9 \pm 3.7$ & $4.4 \pm 2.3$ & $2.4 \pm 2.4$ & $371 \pm 105$ \\
\hline Tree 23 & $2.3 \pm 1.3$ & $0.4 \pm 0.3$ & $0.3 \pm 0.2$ & $4.3 \pm 4.6$ & $5.5 \pm 2.5$ & $2.1 \pm 1.7$ & $433 \pm 108$ \\
\hline Tree 24 & $8.0 \pm 8.9$ & $0.4 \pm 0.1$ & $0.3 \pm 0.3$ & $7.8 \pm 8.6$ & $1.6 \pm 1.5$ & $1.2 \pm 1.6$ & $611 \pm 124$ \\
\hline Tree 25 & $14.6 \pm 7.5$ & $0.7 \pm 0.4$ & $0.2 \pm 0.4$ & $4.9 \pm 5.0$ & $2.5 \pm 1.0$ & $1.1 \pm 0.9$ & $573 \pm 137$ \\
\hline Tree 26 & $19.3 \pm 13.6$ & $0.6 \pm 0.2$ & $0.4 \pm 0.5$ & $6.0 \pm 6.3$ & $2.6 \pm 1.4$ & $1.2 \pm 0.9$ & $618 \pm 140$ \\
\hline Tree 27 & $4.8 \pm 2.5$ & $1.5 \pm 1.0$ & $0.1 \pm 0.1$ & $1.3 \pm 1.9$ & $2.5 \pm 1.4$ & $2.3 \pm 1.9$ & $226 \pm 52$ \\
\hline Tree 28 & $10.8 \pm 5.2$ & $0.3 \pm 0.1$ & $0.3 \pm 0.3$ & $10.1 \pm 8.4$ & $1.1 \pm 0.7$ & $1.1 \pm 1.4$ & $604 \pm 166$ \\
\hline Median & 3.2 & 0.5 & 0.1 & 2.8 & 2.9 & 1.4 & 365 \\
\hline Minimum & 0.3 & 0.1 & 0.0 & 0.0 & 0.1 & 0.0 & 150 \\
\hline Maximum & 40.4 & 2.3 & 5.1 & 19.8 & 9.5 & 5.8 & 810 \\
\hline
\end{tabular}




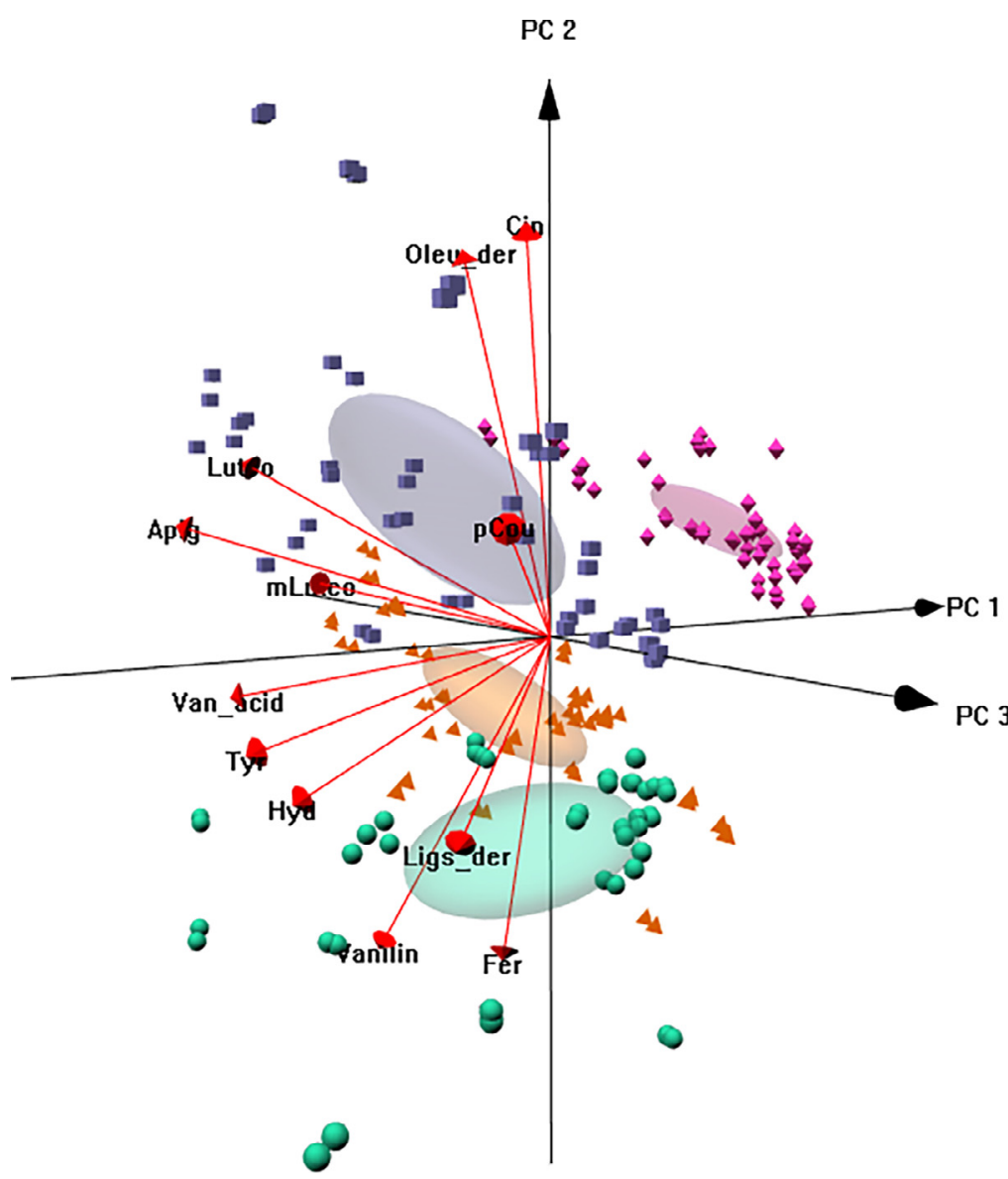

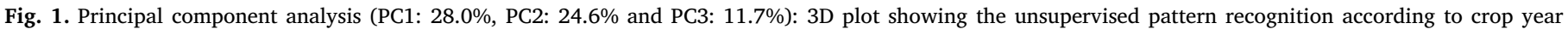
(2014-2017) based on the individual phenolic contents of olive oils obtained from olives collected from centenarian trees.

the enzymatic hydrolysis of oleuropein, but other hydroxytyrosol derivatives are also found, being these the main contributors for the health claim (Torres et al., 2018). However, the content of phenolic compounds in olive oil (both qualitative and quantitative) is strongly affected by diverse factors, giving rise to olive oils with diverse composition and quality over the years (García-Vico et al., 2017). Examples of that are cultivar, geographical origin, irrigation, edapho-climatic conditions, production processes, fruit maturation, harvesting methods, fruit freshness before extraction, extraction method and storage conditions (Dabbou, Chehab, Taticchi, Servili, \& Hammami, 2015; Franco et al., 2014; Gómez-Caravaca, Maggio \& Cerretani, 2016; Köseoğlu, Sevim, \& Kadiroğlu, 2016; Sánchez de Medina, Priego-Capote, \& Castro, 2015; Tovar, Romero, Alegre, Girona, \& Motilva, 2002). Thus, even under the most adequate productive and technological conditions, the same tree can give rise to olive oils with different compositions over the years, being difficult to identify the most adequate cultivars for ensuring a consistent bioactive richness if not studied over a long timeperiod. So far, most of the studies have only been focused on assessing olive oil phenolic composition over short time-periods (one or two years) and very few up to three years, which is not enough to really support the conclusions. On the other hand, in the last decades, there has also been a gradual loss of the genetic heritage potential in olive cultivation worldwide, with a substantial increase of only a minor number of cultivars, mostly based on their agricultural characteristics, particularly the high expected production yields. In a competitive rising marked, the search for innovation and product differentiation, such as the possibility of holding health claims, can only be achieved by reorient the focus to the positive characteristics of the olive oils, rather than the productivity by itself. In this sense, the pursuit for olive oils richer in antioxidant compounds, particularly in phenolic compounds that may support the health claim, can be envisaged through the study and selection of specific olive tree genotypes. Focusing this search on ancient olive trees may also contribute to the future preservation of olive tree cultural heritage.

In this context, this work aimed to study the phenolic composition of olive oils produced from centenarian olive trees, aiming to characterize and possibly select those that enable a consistent production of olive oils richer in polyphenols over the years, contributing to olive oil valorization and probably ensuring health claims. For this, 28 centenarian olive trees, from one Portuguese olive grove, were selected and, during four harvest seasons (2014-2017), the olive oils polyphenol compositions were analyzed.

Unsupervised and supervised statistical techniques were used helping to classify/discriminate olive trees aiming to identify the most promising specimens in terms of health and nutritional claims.

\section{Material and methods}

\subsection{Sampling}

\subsubsection{Tree selection}

The olive trees studied were grown in a centenarian olive grove $(\approx 250$ years) located in the northeast of Portugal, near Mirandela (Suçães, N $41^{\circ}$ 29.425; W $7^{\circ} 15.490$ ). According to our best knowledge, this grove includes the oldest trees of the region, from diverse cultivars most of them unknown. The olive grove has 140 trees and, taking into account the tree appearance, structure and trunk thickness, which are classical indicators of the tree age, 28 distinct olive trees $(20 \%$ of the trees), considered representative of the grove diversity, were selected and individually marked (Fig. S1 of the supplementary material). 


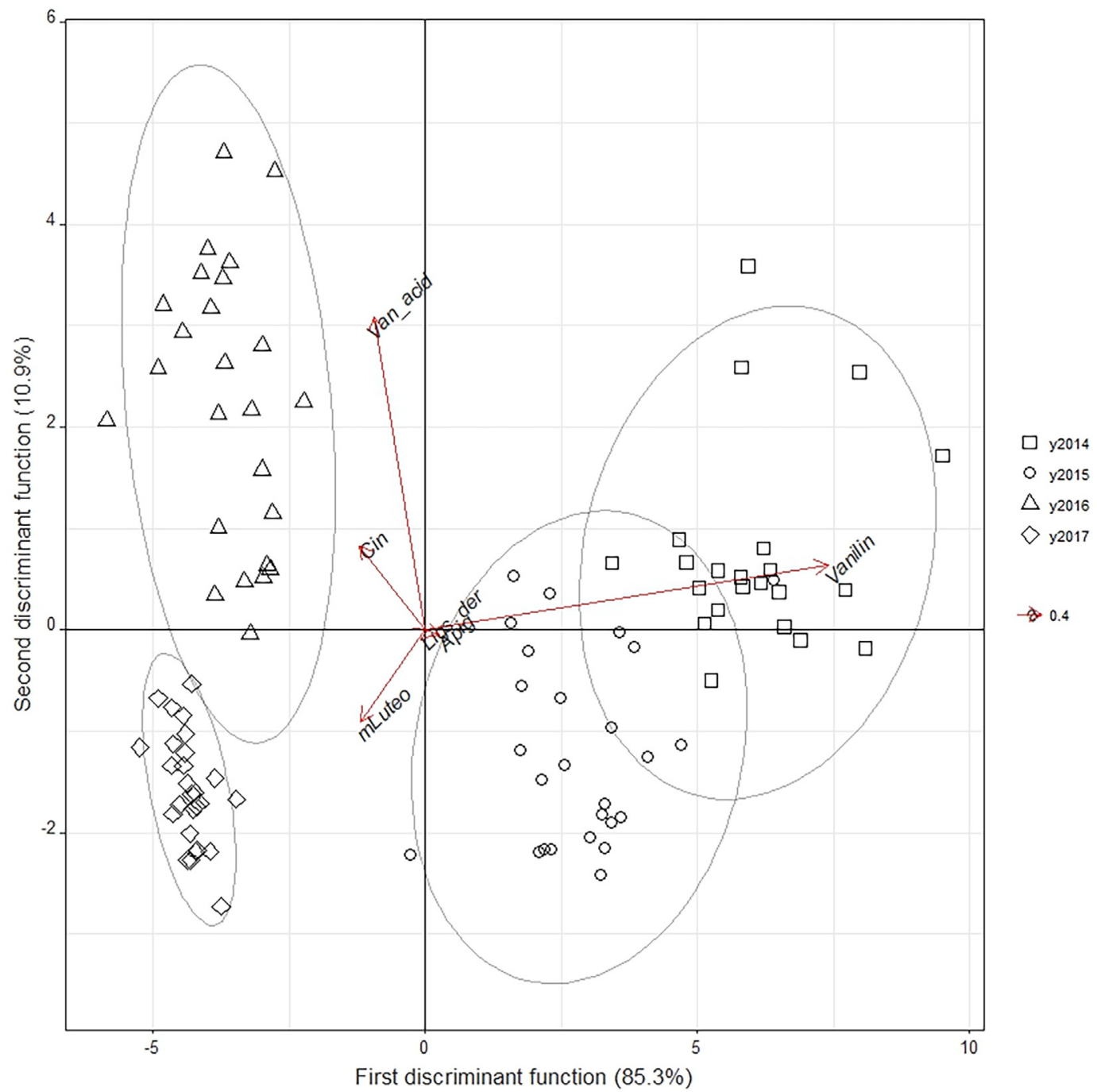

Fig. 2. Linear Discriminant analysis (1st DF: 85.3\%, and 2nd DF: 10.9\%): 2D plot showing the discrimination of olive oil according to the production years based on the individual phenolic contents of oils obtained from olives collected from centenarian trees during four consecutive crop years (2014-2017).

\subsubsection{Harvest}

Along four consecutive crop years (2014-2017), and from each tree, approximately three kilograms of fruits were manually picked. All fruits were visually inspected and the fruits damaged or attacked by pests and diseases were discarded. To avoid the influence of the maturity stage on the olive oil composition, harvest occurred always when the fruits were between the maturity stage (MI) two and three, which corresponds to the fruit epidermis with red spots in less than half of the olive (MI 2) and the fruit epidermis red or purple in more than half of the olive (MI 3) (Hermoso et al., 1991). Thus, every year the harvest occurred during the month of November, namely on the 10th and 11th days in 2014; on the 2nd and 3rd days in 2015; on the 07th and 08th days in 2016; and, on the 13th and 14th days in 2017.

\subsubsection{Oil extraction}

The fruits were processed in the first $24 \mathrm{~h}$ after harvest, in a pilot extraction plant with an Abencor analyzer (Comercial Abengoa S.A., Seville, Spain), with three main units: a mill, a thermobeater where malaxation takes place at controlled temperature, and a centrifuge. Olives were milled, the paste was homogenized, and about $700 \mathrm{~g}$ were transferred to the thermobeater unit $(20 \mathrm{~min})$ for malaxation, using a thermostatic water bath at $25{ }^{\circ} \mathrm{C}$. In the final $5 \mathrm{~min}$ of each malaxation, $100 \mathrm{~mL}$ of water at $25^{\circ} \mathrm{C}$ was added to aid olive oil separation. The mixture was centrifuged, decanted, and the olive oil collected. After that, the oils were prepared for analysis, being filtered (Whatman paper $\mathrm{n}^{\circ} 4$ ) over anhydrous sodium sulfate in order to remove the solid particles and residual water. The olive oils were stored in $125 \mathrm{~mL}$ dark bottles and protected from light exposition, at room temperature. During the four years a total of 112 olive oils were analyzed. All the assays were carried out in triplicate within two months after extraction.

\subsection{Phenolic compounds}

\subsubsection{Extraction}

Phenolic compounds extraction was carried out according to the protocol from the International Olive Council (COI/T.20/Doc No 29/ Rev.1, 2017), with minor modifications. Briefly, $0.4 \mathrm{~g}$ of olive oil were weighed in a $10 \mathrm{~mL}$ tube. An accurate amount of the internal standard solution $(25 \mu \mathrm{L}$; syringic acid at $0.15 \mathrm{mg} / \mathrm{mL}$ in methanol/water $80 / 20$ $(\mathrm{v} / \mathrm{v})$ ) was added, and the sealed tube was vortexed for $30 \mathrm{~s}$. The phenolic compounds were extracted with $2.5 \mathrm{~mL}$ of methanol/water solution $(80: 20, v / v)$, being the solution agitated during $30 \mathrm{sec}$ in a vortex, followed by the addition of hexane $(2.5 \mathrm{~mL})$ for a more clear elimination of the fat, and followed again by vortex mixing for $5 \mathrm{~min}$. Afterwards, the mixture was centrifuged at $5000 \mathrm{rpm}$ for $5 \mathrm{~min}$. The lower phase (hydrophilic) was filtered through a $0.22 \mu \mathrm{m}$ microfilter (PVDF). The solution was then taken to almost dryness under a gentle nitrogen stream $\left(40^{\circ} \mathrm{C}\right)$ and immediately reconstituted with $200 \mu \mathrm{L}$ of methanol, 


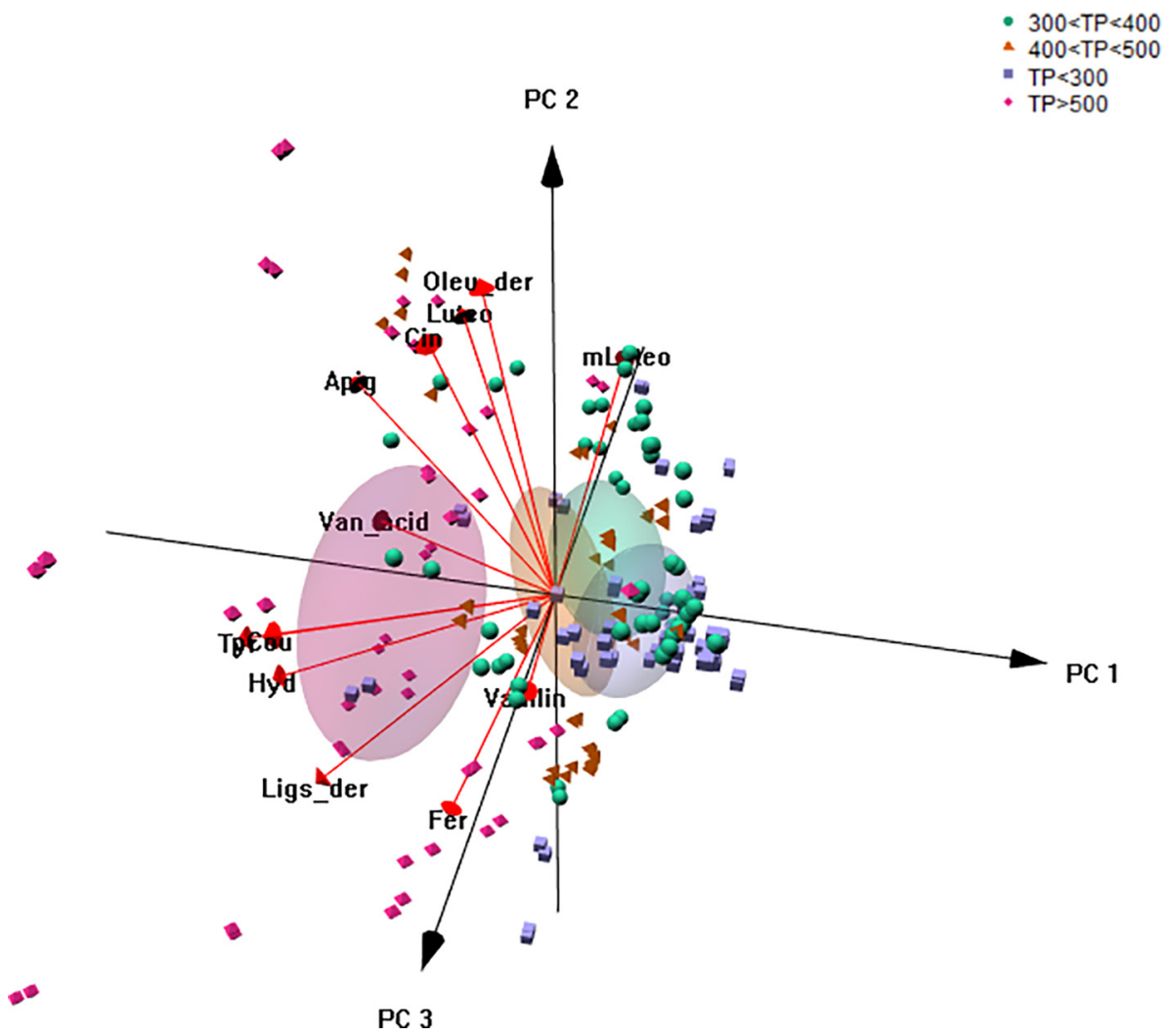

Fig. 3. Principal component analysis (PC1: 28.0\%, PC2: $24.6 \%$ and PC3: $11.7 \%$ ): 3D plot showing the unsupervised pattern recognition according to the predefined groups $\quad(<300 ; \quad 300-400$; 400-500; > $500 \mathrm{mg}$ tyrosol equivalents/kg) of olive oils obtained from olives collected during four consecutive crop seasons (2014-2017) based on their individual phenolic compounds. being ready for injection in the HPLC system. All samples were extracted in duplicate.

\subsubsection{HPLC analysis}

The phenolic composition of the obtained olive oils was evaluated by high performance liquid chromatography (HPLC) with diode array detection (DAD) using an integrated HPLC system from Jasco (Japan) with a data transmitter (LC - NetII/ADC), two integrated pumps (PU 4180), an auto-sampler (AS - 4050), oven (ECOM Eco2000, Czech Republic), and the DAD (MD - 4010). Separation was accomplished on a C18 reversed-phase column (Kinetex C18 $2.6 \mu \mathrm{m} 100 \AA$, $100 \times 3.00 \mathrm{~mm}$, Phenomenex), at $35^{\circ} \mathrm{C}$, using a gradient of water and acetonitrile, both with $0.1 \%$ of formic acid, at a flow rate of $0.8 \mathrm{~mL}$. Peak identification was performed by comparing retention times and UV/Vis spectra $(200-600 \mathrm{~nm}$ ) with those of pure standards (tyrosol, hydroxytyrosol, vanillic acid, ferulic acid, o-coumaric acid, luteolin, cinnamic acid, apigenin, and oleuropein, from diverse suppliers). Secoiridoids tentative identification was oriented by the COI method and available literature. For quantification, UV/Vis detection wavelengths were set to $280 \mathrm{~nm}$ (for simple phenols, vanillic acid, vanillin, lignans and secoiridoids), $325 \mathrm{~nm}$ (for coumaric and ferulic acids), and $365 \mathrm{~nm}$ (for flavonoids). Based on COI, results were expressed as mg of tyrosol equivalents per $\mathrm{kg}$ of olive oil for each individual compound while the total phenols content corresponded to the sum of all individual compounds quantified.

\subsection{Statistical analysis}

The possible effect of crop year (from 2014 to 2017) on the olive oil phenolic profile was evaluated using unsupervised and supervised multivariate pattern recognition techniques namely, principal component analysis (PCA) and linear discriminant analysis (LDA), based on the contents of the individual phenolic compounds. These two chemometric tools were also used to assess the possibility of using the same phenolic profiles to discriminate olive oils after regrouping samples into
4 groups based on their richness in total phenolic compounds $(<300$; 300 to $400 ; 400$ to 500 and $>500 \mathrm{mg}$ tyrosol equivalents $/ \mathrm{kg}$ ), which can be related to possible olive oil health claims. For PCA, the individual phenolic contents were centered and scaled minimizing data variability. LDA was coupled with the meta-heuristic simulated annealing (SA) variable selection algorithm, aiming to identify the phenolic compounds that could enable olive oils discrimination according to the crop year or the phenolic richness (i.e., which were most influenced by each factor), regardless the olive tree from which olives were collected. Indeed, SA algorithm is able to discard redundant variables (i.e., in this case, phenolic compounds), selecting the most influent ones and so, maximizing the correct overall classification percentages (i.e., maximizing the model predictive sensitivity) and minimizing possible noise effects (Bertsimas \& Tsitsiklis, 1992; Cadima, Cerdeira, \& Minhoto, 2004; Kirkpatrick, Gelatt, \& Vecchi, 1983). The predictive performances of the LDA-SA models were evaluated considering the leave-one-out cross-validation (LOO-CV) and the repeated K-fold crossvalidation (repeated K-fold-CV) techniques. In the repeated K-fold-CV, data was randomly split into $\mathrm{K}$ folds, being each of the folds left out in turn for internal-validation and the other K-1 folds used to establish the model. After all folds have been used for validation purposes, the $\mathrm{K}$ estimates are averaged to get the overall resampled estimate (Kirkpatrick et al., 1983). In this work the K-folds were set equal to 4, enabling the random formation of internal-validation subsets with $25 \%$ of the initial data. The procedure was repeated 10 times, which allowed putting the model under stress. The variables were scaled and centered before modeling to normalize the weight of each variable in the final linear classification model. The classification performance of each LDASA model was graphically evaluated by plotting the significant discriminant functions, which would allow visualizing the groups discrimination. Besides, sensitivity values were also calculated to quantitatively assess the discrimination performance. The statistical analysis was performed using the Subselect (Cadima et al., 2004; Cadima, Cerdeira, Silva, \& Minhoto, 2012; Kuhn \& Johnson, 2013) and MASS (Venables \& Ripley, 2002) packages of the open source statistical 


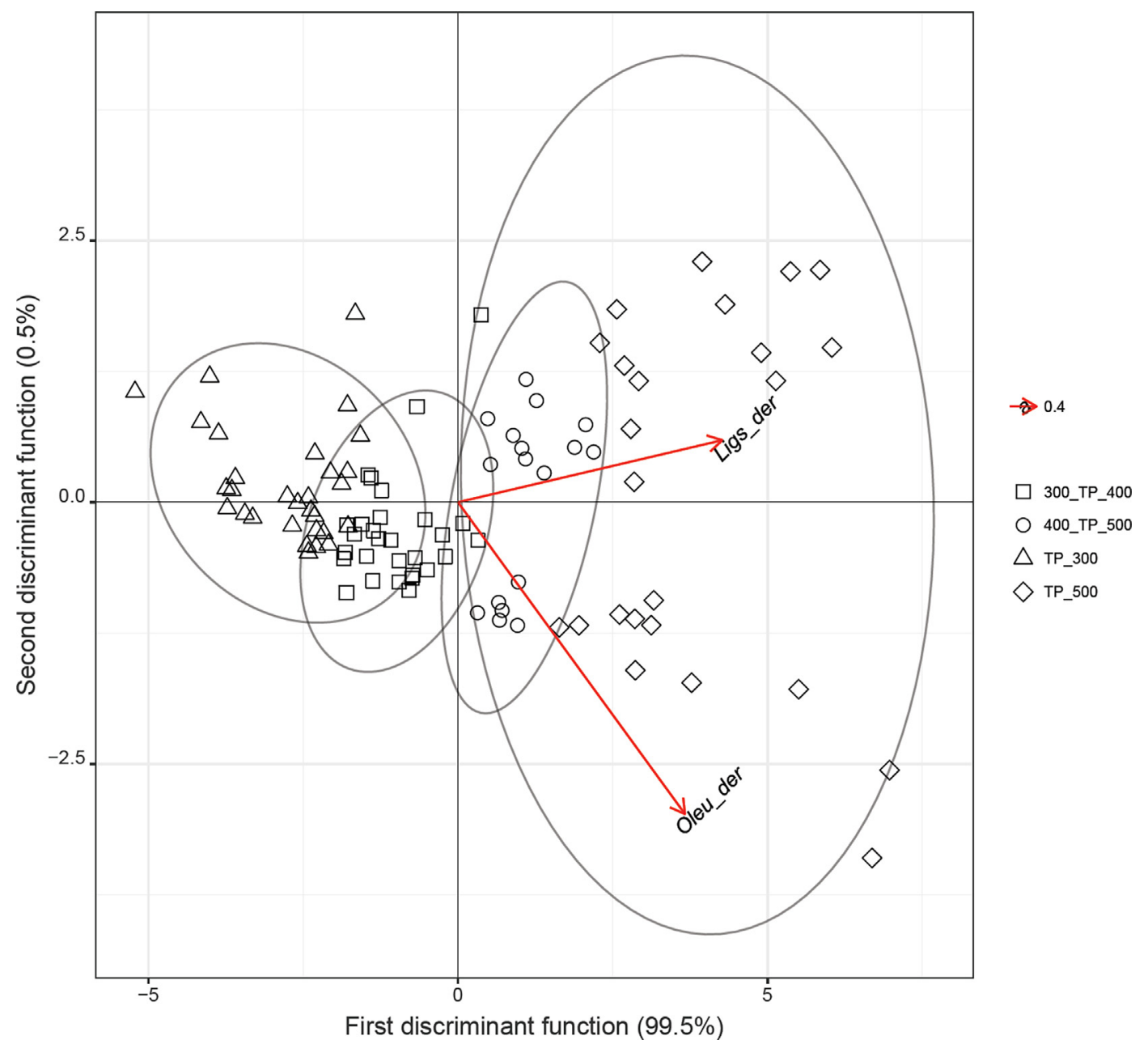

Fig. 4. Linear Discriminant analysis (LDA1: 99.5\%, and LDA2: 0.5\%): 2D plot showing the discrimination of olive oil according to the predefined groups ( $<300$; 300-400; 400-500; > 500 mg tyrosol equivalents/ $\mathrm{kg}$ ) of olive oils obtained from olives collected during four consecutive crop seasons (2014-2017) based on their individual phenolic compounds.

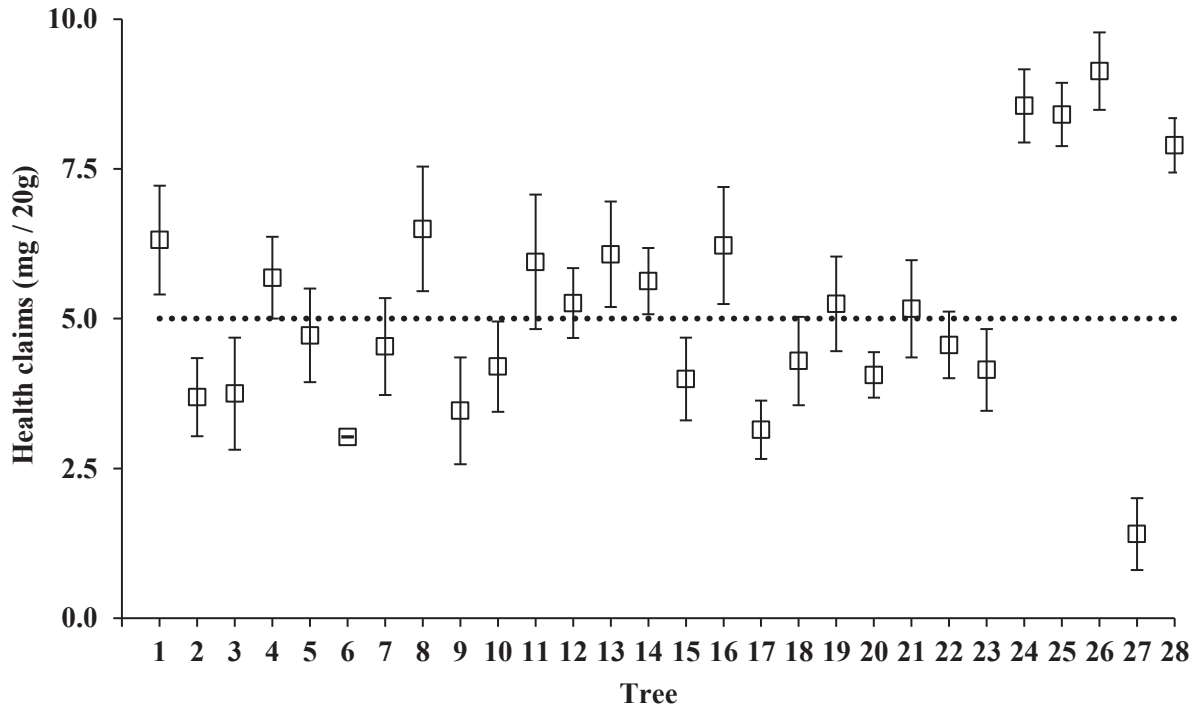

Fig. 5. Fulfilment of the health claim (according to European Regulation EU 432/2012) based on the sum of free tyrosol, free hydroxityrosol and oleuropein derivatives (in $\mathrm{mg}$ of tyrosol equivalents $/ 20 \mathrm{~g}$ olive oil) for the 112 olive oils obtained from each of the selected 28 centenarian trees (mean \pm standard error considering the four consecutive crop years evaluated). 
program R (version 2.15.1), at a 5\% significance level.

\section{Results and discussions}

\subsection{Phenolic compounds identification and quantification}

In the present work, it was studied the phenolic profile of olive oils extracted during four crop years (2014-2017) from 28 individual centenarian olive trees, in a total of 112 olive oil samples, all classified as extra virgin olive oil (data not shown). Table 1 shows the mean contents $(\bar{x})$ and the respective standard deviations $(s d)$ of each phenolic compound detected in the olive oils, which were organized in five phenolic groups, namely: phenolic alcohols (hydroxytyrosol and tyrosol), flavonoids (luteolin, apigenin and methyl luteolin), secoiridoids aglycons (sum of aldehydic form of oleuropein aglycone, dialdehydic and oxidized dialdehydic forms of decarboxymethyl oleuropein aglycone for oleuropein derivatives and sum of oxidised dialdehyde and dialdehyde forms of decarboxymethyl ligstroside aglycone, oxidised aldehyde, dialdehyde and hydroxylic forms of ligstroside aglycone for ligstroside derivatives), phenolic acids ( $o$ - and $p$-coumaric acid, ferulic acid and cinnamic acid) and dihydroxybenzoic derivatives (vanillic acid and vanillin). All the values are within the ranges reported for olive oils (Alowaiesh, Singh, Fang, \& Kailis, 2018; Franco et al., 2014; OlmoGarcía et al., 2017). Nevertheless, the phenolic amount in olive oils are dependent of different factors such as genotype, fruit ripening stage, agro-climatic conditions, production year and geographical origin (Franco et al., 2014; Sánchez de Medina et al., 2015; Tovar et al., 2002). Aware of influence of diverse environmental factors in the olive oil phenolic composition, in the present work, all the trees were grown under the same environmental conditions, subjected to the same agronomic factors, and the fruits were collected at similar maturation indexes.

Among the five phenolic compound groups identified, secoiridoids aglycons were the main group (Table 1 ), which included mainly the dialdehydic form of oleuropein (oleacein; 3,4-DHPEA-DEA), its monoaldehydic form (3,4-DHPEA-EA) and the equivalent ligstroside derivatives (oleocanthal $-p$-HPEA-DEA and $p$-HPEA-EA, respectively). The mean contents for all oleuropein derivatives varied between 32 (tree 27) and $250 \mathrm{mg}$ of tyrosol equivalents/kg of olive oil (tree 24) while those of ligstrodide derivatives varied between 85 (tree 17) and $585 \mathrm{mg}$ of tyrosol equivalents/kg of olive oil (tree 26). Flavonoids were the second group with higher contents (Table 1 ). The most representative compound in this class, apigenin, varied from 2.3 (tree 10) to $16.5 \mathrm{mg}$ of tyrosol equivalents $/ \mathrm{kg}$ of olive oil (tree 13). For the phenolic alcohols group, hydroxytyrosol ranged from 1.1 (tree 27) to $7.1 \mathrm{mg}$ of tyrosol equivalents $/ \mathrm{kg}$ of olive oil (tree 26), while tyrosol from 1.5 (tree 9) to $12.1 \mathrm{mg} / \mathrm{kg}$ of olive oil (tree 26). These low amounts are in accordance with the freshness of the extracted olive oils, as they increase with time by hydrolysis of secoiridoids. The group of phenolic acids included $o$ and $p$-coumaric, ferulic, and cinnamic acids; whereas in the group of dihydroxybenzoic acids two compounds were identified (vanillic acid and vanillin) (Table 1). Similar compounds and amounts were reported by Peres et al. (2016) in olive oils from the Portuguese varieties Galega Vulgar and Cobrançosa extracted at early ripening stages, by Veneziani et al. (2018) for olive oils from six Italian cultivars (Frantoio, Leccino, Gentile, Ogliarola garganica, Moraiolo and San Felice) and by Kotsiou and Tasioula-Margari (2016) in Greek extra-virgin olive oils. The highest total phenolic contents were observed for the trees 24, 26 and 28 with average values for the four years of 611,618 and $604 \mathrm{mg}$ of tyrosol equivalents/kg of olive oil, respectively. On the contrary, the lowest contents were consistently obtained for the olive oils extracted from the trees 2, 6, 9 and 27, with values of 279, 285, 271 and $226 \mathrm{mg}$ of tyrosol equivalents $/ \mathrm{kg}$ of olive oil, respectively (Table 1 ). These results show that some of the trees (e.g., 24, 26 and 28) could be good candidates for multiplication and for breeding programs if the aim is to obtain olive oils with high levels of total phenolic compounds. As mentioned before, the phenolic contents in olive oil is affected by several factors such as genotype, maturation, geographic origin and olive genotype (Aparicio-Ruiz et al., 2016; Caruso et al., 2014; Veneziani et al., 2018). However, in the present work, since all trees were grown under the same conditions, the genotype is the main varying factor, suggesting that, as mentioned by Valls et al. (2015), the production of phenolic compounds is genetically regulated.

\subsection{Effect of crop year on the phenolic composition}

As previously stated, the phenolic profile of olive oil is also dependent of environmental conditions (Gómez-Caravaca et al. (2016), and this is among the less studied factors in the literature, due to the time required for reaching soundness conclusions. Thus, the influence of the crop year on the amounts of individual phenolic compounds found in the olive oils obtained from ancient olive trees during four consecutive crops was statistically evaluated. As can be seen from Fig. 1, based on the olive oil's individual phenolic contents (Table 1), PCA allowed the unsupervised discrimination of the oils produced from the 28 olive trees according to the crop year, showing that the production year had a high influence on the phenolic fraction content. The first 3 principal component (PC) functions explained more than $64 \%$ of the data variability $(28.0 \%, 24.6 \%$ and $11.7 \%$ for PC1, PC2 and PC3, respectively). The results also pointed out that ferulic acid, ligstroside derivatives and vanillin were the phenolic compounds that mostly contributed to discriminate olive oils produced in 2014. Regarding the year 2015, hydroxytyrosol, tyrosol and vanillic acid were those that most influenced the olive oil phenolic composition while in 2016, cinnamic acid, luteolin, $p$-coumaric acid and oleuropein derivatives were leading compounds in the discrimination. Finally, for 2017, all phenolic compounds were similarly influenced. These results are in agreement with the findings of Köseoğlu et al. (2016) which reported that the agro-climatic conditions influence the phenolic composition of olive oils. Our data showed that the different trends observed over the years have a strong influence of the climatic conditions (Supplementary material, Fig. S2), particularly the water amounts over the year since the temperatures ranges were quite similar. Also, the prominence of both free hydroxityrosol and tyrosol in 2015 , seems to be attributed to an increased hydrolysis of secoiridoids. This year was characterized by an accelerated maturation, as can be depicted by the shorter harvest date to attain similar maturation degrees, and October was characterized by an intense rainfall. Both factors could have contributed to this phenomenon. The clear distinction of the 2017 phenolic pattern, with lower amounts of all compounds, can be attributed to the severe water shortage observed in the region (Fig. S2). Although climatic stress is recognized as a favorable factor for an increased phenolic synthesis (Malheiro, Rodrigues \& Pereira, 2015), the water shortage observed in that year at the budding process could probably had a negative influence in the amount of phenolic compounds in the fat, a situation that worth being explored in future studies.

Finally, LDA-SA was also applied to verify which of the phenolic compounds were more influenced by the crop year, by reducing the number of non-redundant variables. A LDA-SA model, with three significant discriminant functions (explaining $85.3 \%, 10.9 \%$ and $3.8 \%$ of the data variability, respectively) was established based on the experimental contents of 6 phenolic compounds (importance of contribution: vanillin $>$ vannillic acid $>$ methyl luteolin $>$ cinnamic acid $>$ apigenin $\approx$ ligstroside derivatives). The model allowed the correct classification of $96 \%$ of the original grouped data (Fig. 2), $96 \%$ for the LOO-CV ( 4 of the 100 olive oils misclassified according to the crop year) and $94 \pm 4 \%$ for the repeated K-fold-CV ( 4 folds $\times 10$ repeats leading to 40 randomly runs, with sensitivities ranging from $79 \%$ to $100 \%$ ). From Fig. 2 it can be stated that vanillin was the phenolic compound that mostly contributed for the differentiation of the olive oils obtained in 2014 and in 2015. On the contrary, cinnamic acid and vanillic acid were those that mostly contributed to the discrimination of 
the olive oils produced in 2016. Finally, for 2017, methyl luteolin was the phenolic compound that had the highest influence but the negative values on both components are in agreement with the previous observation of lower amounts of all phenolic compounds in that year. These results showed the accuracy of the established multivariate linear classification approach, showing that the contents of the 6 abovementioned phenolic compounds could be used as chemical markers to discriminate olive oils according to crop year, confirming that the production year significantly influences the phenolic composition and nutritional quality of the olive oil. Additionally, it is important to stress that the most important phenolic compounds from the health claim point of view, that is, tyrosol, hydroxytyrosol and oleuropein derivatives presented a low inter-year variability within the same trees.

\subsection{Olive oil discrimination according to phenolic classes and indirect tree classification}

For a better identification of the olive trees with the highest phenolic potential, the 112 olive oils were split into 4 different groups, regardless the crop year, according to the sum of all quantified phenolic compounds (total phenolics - TP), namely TP $<300 \mathrm{mg} / \mathrm{kg}$ of olive oil, $300 \leq \mathrm{TP}<400 \mathrm{mg} / \mathrm{kg}$ of olive oil, $400 \leq \mathrm{TP}<500 \mathrm{mg} / \mathrm{kg}$ of olive oil and TP $\geq 500 \mathrm{mg} / \mathrm{kg}$ of olive oil, all in tyrosol equivalents. The PCA on the individual phenolic compounds showed that the profiles allowed a satisfactory unsupervised differentiation of the oils according to the TP groups (Fig. 3). The results pointed out that, although 4 groups were proposed, the most evident differentiation was from olive oils with a TP greater than $500 \mathrm{mg} / \mathrm{kg}$ of olive oil from the others, being the vanillic acid, hydroxytyrosol, tyrosol, $p$-coumaric acid and ligstroside derivatives the phenolic compounds that most influenced this unsupervised discrimination. The LDA-SA procedure allowed establishing a model with two discriminant functions (explaining $99.5 \%$ and $0.5 \%$ of the data variability, respectively) based on the contents of oleuropein derivatives and ligstroside derivatives. The classification model had sensitivities of $93 \%$ of the original grouped data (Fig. 4), 92\% for the LOO-CV (4 of the 100 olive oils misclassified according to the crop year) and $91 \pm 6 \%$ for the repeated K-fold-CV ( 4 folds $\times 10$ repeats leading to 40 randomly runs, with sensitivities ranging from $79 \%$ to $100 \%)$. As can be inferred from Fig. 4, the olive oil groups are located along the 1st discriminant function, and seem to be directly correlated (i.e., higher values of the 1st discriminant function corresponded to olive oils with higher overall phenolic contents). The overall results pointed out that, regardless the olive tree and the crop year, the TP content is mostly influenced by the individual secoiridoids aglycons, quantitative the most important phenolic group in the olive oils.

\subsection{Health claims}

According to EU Regulation 432/2012 (2012) and EFSA (2012), if an olive oil has a minimum of $5 \mathrm{mg}$ of hydroxytyrosol and its derivatives (e.g. oleuropein complex and tyrosol) per $20 \mathrm{~g}$ of olive oil, the claim of "Olive oil polyphenols contribute to the protection of blood lipids from oxidative stress" could be used in the label. In this regard, it is worth mentioning that this classification is ambiguous, without a clear definition of what phenolic compounds should be included in the claim (Tsimidou \& Boskou, 2015). Using the most conservative approach, that is, solely the sum of free tyrosol, free hydroxytyrosol and hydroxytyrosol derivatives (oleuropein derivatives and hydroxytyrosol acetate), Fig. 5 shows the mean amounts and standard deviation for the olive oils obtained from each tree in the four consecutive crop years studied per $20 \mathrm{~g}$ of olive oil, as required by the health claim. The average values varied from $1.4 \mathrm{mg} / 20 \mathrm{~g}$ of olive oil (tree 27) to $9.1 \mathrm{mg}$ / $20 \mathrm{~g}$ of olive oil (tree 26), with very similar dispersion between most of the trees. Considering all trees evaluated, it was observed that $50 \%$ of them (14 trees) led to olive oils with levels higher than the minimum required for fulfilling the health claim. The olive oils obtained from those trees (tree $\mathrm{n}^{\circ} 1,4,8,11-14,16,19,21,24-26$ and 28) could be labeled as "Olive oil polyphenols contribute to the protection of blood lipids from oxidative stress". In particular, olive oils obtained from three of these trees, namely trees $n^{\circ} 24,25$ and 26 , contained consistently through the four years at least $50 \%$ more than the minimum required, representing good candidates for selection. On the contrary, trees $n^{\circ} 6$, 17 and 27, showed the lowest amounts of "hydroxytyrosol + tyrosol + oleuropein derivatives" (Fig. 5). Apart from the health benefits, the total phenolic amounts may also influence the olive oil quality evolution during storage, being known that olive oils rich in phenolic compounds possess greater shelf lives due to their higher resistance to oxidation.

\section{Conclusions}

With this work it was possible to clarify that the year of production is one of the main factor influencing the phenolic profile of olive oils, even if produced under the same agro-climatic conditions and extracted under the most adequate technological conditions. Therefore, for effective conclusions on the potential of certain specimens or cultivars for producing olive oil consistently rich in antioxidants it is necessary to study then over a huge period range. From the 28 specimens of centenarian trees selected for this study it was possible to identify specimens that gave olive oils with exceptional high content of phenolic compounds, consistent through different crop years, while other had no clear interest in this regard. These selected trees are potential candidates for breeding in order to obtain differentiated oils that could hold a health claim, promoting also to the future preservation of the genetic heritage.

\section{Acknowledgements}

This work was financially supported by Strategic Project PEst-OE/ AGR/UI0690/2014 (CIMO), Project UID/QUI/50006/2013 (REQUIMTE-LAQV); and Project POCI-01-0145-FEDER-006984 (Associate Laboratory LSRE-LCM); all funded by Fundo Europeu de Desenvolvimento Regional (FEDER) through COMPETE2020-Programa Operacional Competitividade e Internacionalização (POCI) and by national funds through Fundação para a Ciência e a Tecnologia (FCT), Portugal. This work was also supported by PRODER project OliveOld Identificação e caraterização de oliveiras centenárias para obtenção de produtos diferenciados" $n^{\circ}$ 53988. Nuno Rodrigues thanks FCT, POPHQREN and FSE for the Ph.D. Grant (SFRH/BD/104038/2014).

\section{Appendix A. Supplementary data}

Supplementary data to this article can be found online at https:// doi.org/10.1016/j.foodchem.2018.09.106.

\section{References}

Alowaiesh, B., Singh, Z., Fang, Z., \& Kailis, S. G. (2018). Harvest time impacts the fatty acid compositions, phenolic compounds and sensory attributes of Frantoio and Manzanilla olive oil. Scientia Horticulturae, 234, 74-80.

Aparicio-Ruiz, R., García-González, D. L., Oliver-Pozo, C., Tena, N., Morales, M. T., \& Aparicio, R. (2016). Phenolic profile of virgin olive oils with and without sensory defects: Oils with non-oxidative defects exhibit a considerable concentration of phenols. European Journal of Lipid Science and Technology, 118, 299-307.

Bertsimas, D., \& Tsitsiklis, J. (1992). Simulated annealing. Statistical Science, 8, 10-15. Cadima, J., Cerdeira, J. O., \& Minhoto, M. (2004). Computational aspects of algorithms for variable selection in the context of principal components. Computational Statistics \& Data Analysis, 47, 225-236.

Cadima, J., Cerdeira, J.O., Silva, P.D., \& Minhoto, M. (2012). The subselect R package. http://cran.rproject.org/web/packages/subselect/vignettes/subselect.pdf. Accessed $15 / 02 / 2016$

Caruso, T., Marra, F. P., Costa, F., Campisi, G., Macaluso, L., \& Marchese, A. (2014). Genetic diversity and clonal variation within the main Sicilian olive cultivars based on morphological traits and microsatellite markers. Scientia Horticulturae, 180, on morphol 138 .

Cicerale, S., Lucas, L., \& Keast, R. S. (2010). Biological activities of phenolic compounds 
present in virgin olive oil. International Journal of Molecular Science, 11, 458-479.

Dabbou, S., Chehab, H., Taticchi, A., Servili, M., \& Hammami, M. E. (2015). Content of fatty acids and phenolics in Coratina olive oil from Tunisia: Influence of irrigation and ripening. Chemistry \& Biodiversity, 12, 397-406.

EFSA (2012). NDA. Panel, scientific opinion on the substantiation of a health claim related to polyphenols in olive and maintenance of normal blood HDL cholesterol concentrations (ID 1639, further assessment) pursuant to Article 13(1) of Regulation (EC) No 1924/2006. European Food Safety Authority Journal, 10.

European Regulation EU 432/2012 "Establishing a list of permitted health claims made on foods, other than those referring to the reduction of disease risk and to children's development and health". Official Journal of the European Union, L136, 2012, 1-40.

Fernández, E., Vidal, L., \& Canals, A. (2018). Rapid determination of hydrophilic phenols in olive oil by vortex-assisted reversed-phase dispersive liquid-liquid microextraction and screen-printed carbon electrodes. Talanta, 181, 44-51.

Franco, M. N., Galeano-Díaz, T., López, O., Fernández-Bolaños, J. G., Sánchez, J., De Miguel, C., et al. (2014). Phenolic compounds and antioxidant capacity of virgin olive oil. Food Chemistry, 163, 289-298.

Fregapane, G., \& Salvador, M. D. (2013). Production of superior quality extra virgin olive oil modulating the content and profile of its minor components. Food Research International, 54, 1907-1914.

García-Rodríguez, R., Romero-Segura, C., Sanz, C., \& Pérez, A. G. (2015). Modulating oxidoreductase activity modifies the phenolic content of virgin olive oil. Food Chemistry, 171, 364-369.

García-Vico, L., García-Rodríguez, R., Sanz, C., \& Pérez, A. G. (2017). Biochemical aspects of olive freezing-damage: Impact on the phenolic and volatile profiles of virgin olive oil. LWT - Food Science and Technology, 86, 240-246.

García-Villalba, R., Carrasco-Pancorbo, A., Oliveras-Ferraros, C., Vázquez-Martín, A. Menéndez, J. A., Segura-Carretero, A., et al. (2010). Characterization and quantification of phenolic compounds of extra-virgin olive oils with anticancer properties by a rapid and resolutive LC-ESI-TOF MS method. Journal of Pharmaceutical and Biomedical Analysis, 51, 416-429.

Gómez-Caravaca, A. M., Maggio, R. M., \& Cerretani, L. (2016). Chemometric applications to assess quality and critical parameters of virgin and extra-virgin olive oil. A review. Analytica Chimica Acta, 913, 1-21.

Hermoso, M., Uceda, M., García, A., Morales, B., Frias, M.L., \& Fernandez, A. (1991) Elaboración de Aceite de Calidad; Consejeria de Agricultura y Pesca, Serie Apuntes 5/ 92; Sevilla, Spain.

IOC, International Olive Council, Chemical analysis of olive oils, Method-Determination of Biophenols in olive oil by HPLC, COI/T.20/Doc No 29/Rev.1 2017.

Khoddami, A., Wilkes, M. A., \& Roberts, T. H. (2013). Techniques for analysis of plant phenolic compounds. Molecules, 18, 2328-2375.

Khymenets, O., Farré, M., Pujadas, M., Ortiz, E., Joglar, J., Covas, M. I., \& Torre, R. (2011). Direct analysis of glucuronidated metabolites of main olive oil phenols in human urine after dietary consumption of virgin olive oil. Food Chemistry, 126, 306-314.

Kirkpatrick, S., Gelatt, C. D., \& Vecchi, M. P. (1983). Optimization by simulated annealing. Science, 220, 671-680.

Köseoğlu, O., Sevim, D., \& Kadiroğlu, P. (2016). Quality characteristics and antioxidant properties of Turkish monovarietal olive oils regarding stages of olive ripening. Food Chemistry, 212, 628-634.

Kotsiou, K., \& Tasioula-Margari, M. (2016). Monitoring the phenolic compounds of Greek extra-virgin olive oils during storage. Food Chemistry, 200, 255-262.
Kuhn, M., \& Johnson, K. (2013). Applied predictive modeling. New York: Springer Science Business Media.

Malheiro, R., Rodrigues, N., \& Pereira, J. A. (2015). Olive oil phenolic composition as affected by geographic origin, olive cultivar and cultivation systems. In Olive and olive oil bioactive constituents Ed. Dimitrios Boskou. Cap. 4, AOCS Press. ISBN 978-1630670-41-2. 30pp.

Olmo-García, L., Bajoub, A., Monasterio, R. P., Fernández-Gutiérrez, A., \& CarrascoPancorbo, A. (2017). Metabolic profiling approach to determine phenolic compounds of virgin olive oil by direct injection and liquid chromatography coupled to mass spectrometry. Food Chemistry, 231, 374-385.

Peres, F., Martins, L. L., Mourato, M., Vitorino, C., Antunes, P., \& Ferreira-Dias, S. (2016). Phenolic compounds of 'Galega Vulgar' and 'Cobrançosa' olive oils along early ripening stages. Food Chemistry, 21, 51-58.

Polari, J. J., Garcí-Aguirre, D., Olmo-García, L., Carrasco-Pancorbo, A., \& Wanga, S. C. (2018). Impact of industrial hammer mill rotor speed on extraction efficiency and quality of extra virgin olive oil. Food Chemistry, 242, 362-368.

Reboredo-Rodríguez, P., Figueiredo-González, M., González-Barreiro, C., Simal-Gándara, J., Salvador, M. D., Cancho-Grande, B., et al. (2017). State of the art on functional virgin olive oils enriched with bioactive compounds and their properties. International Journal of Molecular Sciences, 18, 668.

Sánchez de Medina, V., Priego-Capote, F., \& Castro, M. D. L. (2015). Characterization of monovarietal virgin olive oils by phenols profiling. Talanta, 132, 424-432.

Shahidi, F., \& Ambigaipalan, P. (2015). Phenolics and polyphenolics in foods, beverages and spices: Antioxidant activity and health effects. Journal of Functional Foods, 18, $820-897$.

Torres, A., Espínola, F., Moya, M., Alcalá, S., Vidal, A. M., \& Castro, E. (2018). Assessment of phenolic compounds in virgin olive oil by response surface methodology with particular focus on flavonoids and lignans. LWT - Food Science and Technology, 90, 22-30.

Tovar, M. J., Romero, M. P., Alegre, S., Girona, J., \& Motilva, M. J. (2002). Composition and organoleptic characteristics of oil from Arbequina olive (Olea europaea L.) trees under deficit irrigation. Journal of the Science of Food and Agriculture, 82, 1755-1763.

Tsimidou, M. Z., \& Boskou, D. (2015). The health claim on "olive oil polyphenols" and the need for meaningful terminology and effective analytical protocols. European Journal of Lipid Science and Technology, 117, 1091-1094.

Valls, R., Farràs, M., Suárez, M., Fernández-Castillejo, S., Fitó, M., Konstantinidou, V., et al. (2015). Effects of functional olive oil enriched with its own phenolic compounds on endothelial function in hypertensive patients. A randomised controlled trial. Food Chemistry, 167, 30-35.

Venables, W. N., \& Ripley, B. D. (2002). Modern applied statistics with S (statistics and computing) (4th ed.). New York: Springer.

Veneziani, G., Esposto, S., Taticchi, A., Urbani, S., Selvaggini, R., Sordini, B., et al. (2018) Characterization of phenolic and volatile composition of extra virgin olive oil extracted from six Italian cultivars using a cooling treatment of olive paste. LWT - Food Science and Technology, 87, 523-528.

Visioli, F. (2012). Olive oil phenolics: Where do we stand? Where should we go? Journal of the Science and Food Agriculture, 92, 2017-2019.

Visioli, F., \& Bernardini, E. (2013). Extra virgin olive oil's polyphenols: Biological activities. Current Pharmaceutical Design, 17, 786-804.

Vitaglione, P., Savarese, M., Paduano, A., Scalfi, L., Fogliano, V., \& Sacchi, R. (2015). Healthy virgin olive oil: A matter of bitterness. Critical reviews. Food Science and Nutrition, 55, 1808-1818. 